\title{
Deep Venous Thrombosis and Pulmonary Embolism
}

\section{Prediction, Prevention and Treatment*}

\author{
William W. Coon, m.D. and Park W. Willis, iII, M.D.
}

Ann Arbor, Michigan

$\mathrm{N}^{\mathrm{s}}$ EITHER the frequency nor the importance of the debilitating and fatal consequences of thrombcembolic disease has been fully appreciated by most physicians. The following data are presented to stress the high frequency of this disease, the serious and important nature of its associated sequelae and the difficulties encountered in making the clinical diagnosis. Deep renous thrombosis and pulmonary embolism are disorders of major significance and are responsible for much long term disability. and an appreciable mortality. If there is to be a real reduction in the incidence of pulmonary embolism and the postphlebitic syndrome of edema, induration and ulceration, there must be a greater awareness on the part of the clinician of the many and varied manifestations of thromboembolism, of the value of prompt and effective treatment and of procedures for prevention.

Extrapolation of data obtained at our hospital to mortality statistics of the United States suggests that there may be as many as 47,000 deaths annually in the Cnited States in which pulmonary embolism is the sole cause; in addition, there may be three times this many deaths in which pulmonary embolism has at least played a significant role in the outcome.

There are no good mass surveys on the incidence of ulcers of the leg among the population of the United States. Boyd et al. ${ }^{1}$ suggest that in England it is about five per thousand population. If this figure can be applied to our population, there may be as many as 850,000 people in the United States with ulceration of the leg, the majority secondary to postphlebitic change. 'I he incidence of the other manifestations of the postphlebitic syndrome must be considerably higher than this.

A number of studies have shown that prompt diagnosis and adequate treatment of deep venous thrombosis can appreciably lower the incidence of embolic mortality and the significant disability associated with postphlebitic sequelae. Particularly with regard to pulmonary embolism, there is a real need to achieve a much higher diagnostic rate if one hopes to lower significantly the mortality rate from this complication. A review of the records of large autopsy series at our hospital ${ }^{2}$ has shown that in only 10.6 per cent of patients with proved pulmonary cmboli was a clinical diagnosis of venous thrombosis made prior to death. In another 8.6 per cent of these patients localizing signs, which should have resulted in a diagnosis of deep venous thrombosis of the leg, were present. The difficulties in diagnosis of this entity are illustrated by the fact that in the remaining patients $(80.8$ per cent) there were no signs or symptoms suggesting any thrombotic process in the veins of the leg, despite the fact that a maximum of less than 10 per cent of all emboli could have arisen from a cardiac source.

The repeated demonstration of a high failure rate in the clinical diagnosis of thromboembolic disease precludes the presentation of accurate figures on the incidence of this disease in a hospital population. Estimates based on the constant but rather low clinical diagnosis rate as proved at autopsy, plus prior figures obtained in clinically diagnosed cases of thrombeembolism at our hospital, suggest that as many as 6 per cent of hospitalized patients have deep renous thrombosis, largely undiagnosed, during

* From the Departments of Surgery and Medicinc, University of Michigan Medical School, Ann Arbor. Michigan. 
their hospital stay. (This is admittedly only a gross estimate; it is based on reasonably accurate data obtained from autopsy records, the percentage of cases diagnosed clinically, ratios between the incidence of deep venous thrombosis and fatal embolism, elc. However, this figure has been derived by several different technics, and the approximate incidence of 6 per cent has been obtained in each instance.)

\section{Cinigal Manifestations of Thromboembolic Disease}

Deep Vonous Thrombosis: We strongly believe that there is no justification for the clinical differentiation of the aseptic form of deep venous thrombosis into "phlebothrombosis" and "thrombophlebitis." These terms, in our opinion, represent different phases of the same disease. Organization of a thrombus in any vessel is associated with a sterile inflammatory reaction and round cell infiltration. The manifestations attributed to "thrombophlebitis" merely represent an advanced stage of a thrombotic process which is sufficiently extensive and severe so that deep-lying pathologic changes are reflected in superficial signs. Our studies have shown that it is fallacious to maintain that patients with "thrombophlebitis" are secure from pulmonary embolism; pulmonary emboli develop in a number of patients with the classic signs of thrombophlebitis. Although certain portions of a thrombus may be firmly attached to the vein wall, the propagating proximal tail of the thrombus is usually "free floating" and may easily fragment and result in pulmonary embolism. In addition, there is no histologic basis for distinguishing these entities. We have sectioned a number of thrombosed veins; one area will demonstrate round cell infiltration of the vein wall (supposedly characteristic of thrombophlebitis) while another section through the same thrombus a few millimeters away will show no sign of sterile inflammatory change in the vein wall.

Informately, the majority of episodes of deep venous thrombosis are not associated with any signs or symptoms. All too frequently the first indication of venous thrombosis of the leg is the sudden death of the patient from fatal pulmonary embolism. In our series, even though the presence of iliac or caval thrombi was proved at autopsy, only half the patients had associated clinical signs, and many of these demonstrated only minimal unilateral edema. Complete but localized occlusion of the iliac vein will not produce edema if there is an adcquate number of collaterat channels for the return of venous blood. It is only when there is occlusion of many collateral channels as well that edema is present.

The signs and symptoms of deep venous thrombosis may be vague or totally non-existent. Rarely, plantar or calf pain will tse present. It is usually dull and aching in character, but occasionally, patients will complain of true cramps in the calf of the leg. When pain is present, there is frequenty an associated tenderness along some portion of the course of the deep venous system, in the plantar region of the foot, between the bellies of the gastrocnemius, in the popliteal area, along the femoral canal or in the lower part of the abdomen over the cours: of the iliac veins. local heat and redness may be noted in rare instances. Fever and tachycardia may be suggestive findings, but frequently these manifestations mat he explained away as due to other causes. In the usual casc, even if present, these signs are of little diagnostic value.

Much more frequently, the only clinical sign suggesting the presence of a thrombus occluding the decp venous sytem is edema. Unilateral edema of the leg or asymmetrical bilateral edema should be considered as diagnostic of deep venous thrombosis unless another cause can be definitely proved. Lnfortunately, because of the frequent association of congestive licart failure and thromboembolism, apparently equal edema of both legs will often be present to confuse the clinical picture. In this situation, however, edema is often more marked in the leg involved.

The Septic Form of Deep I'nous Thrombosis ("Septic Thrombophlebilis"): Since the advent of antibiotic theraps, this form of thromboembolic disease is rare. Fever is frequently higher than that seen with aseptic venous thrombosis, but there are no specific diagnostic signs. Such a process usually occurs in association with pelvic inflammatory disease which may result from operations on pelvic structures, childbirth, abortions, appendiceal abscess, etc. Pelvic examination may reveal some tenderness, local heat, fluctuation or induration in the broad ligaments or along the pelvic floor. Fever is usually of longer duration than in the aseptic form of this disease. Not infrequently, the diagnosis becomes apparent only after septic. emboli have produced septic infarcts and secondary abscesses of the lung. Diagnosis is deperdent on the demonstration of some scptic process in close relationship with a portion of the deep venous system. 
"Trousseau Syndrome" ("Migratory Thrombophlehitis"): 'Thrombosis involving either superficial or deep venous segments in more than one area, either concurrently or separated by considerable periods of time, deserves special attention. A history of spontaneously developing thrombosis in superficial veins of the am, abdomen or leg, frequently linked with venous thrombosis of the deep veins of the leg, warrants careful diagnostic work-up. The following clisease cotities most frequently associated with this picture are those which Trousseau originally linked with migratory thrombophlebitis: (1) thromboangiitis obliterans; (2) polycythemia rubra vera; (3) hidden carcinoma, especially of the stomach, pancreas or lung; and (4) collagen disease.

Deep Lenous Thromhosis zeith Significant Ateriosprasm (Phlegmasia Conulea Dolens): Almost all cases of massive venous occlusion of the leg are associated with some degree of artcriospasm. One of the synonyms for iliofemoral venou: thrombosis is "phlegmasia alba dolens." indicating the pallor of the leg thought to be due to spasm of the arteries accompanying the sterile inflammation of the vein. When venous thrombosis of the leg and arteriospasm are of such a degree that cyanosis (cerulea - bluc) is present, a serious situation exists, and the viability of the leg may be in question. It is essential that this entity be recognized promptly and differentiated from primary arterial thrombosis or arterial cmbolism, since the approach to treatment is somewhat different. A pale cold leg with obvious signs of arterial insufficiency may present a difficult diagnostic problem; fortunately, in the usual case of phlegmasia cerulea dolens. marked edema of the leg is present. Such a finding associated with signs of acute arterial insufficiency should suggest the presence of a thrombus involving the venous system. One should not expect to see edema of the leg in the early phase of prinary arterial obstruction; secondary venous occlusion may occur after a period of complete arterial occlusion, but it is not an carly finding. Rarely, massive venous occlusion and the associated arteriospasm may occur so rapidly that there is no time for edema to devclop. In these instances, we believe that rapid institution of specific treatment for phlegmasia cerulea dolens (continuous epidural ssmpathetic block and intravenous administration of heparin) is imperative. These measures will not interfere with subsequent therapy if it is later decided that the primary abnormality is arterial thrombosis or cmbolism. Arteriospasm will usually be promptly relieved by this regimen. If arteriospasm remains unrecognized for a considerable period of time, secondary arterial thrombosis may supervene, and the venous thrombusis becomes more cxtensive. In these cases, the prognosis for survival of the leg is poor; arterial thrombectomy is of little value since massive venous occlusion usually prevents the re-establishment of adequate arterial outflow.

Pulmonary Embolism: All too frequently the first clinical sign of a thrombus in the leg or pelvic reins is one of the cardiorespiratory manifestations of pulmonary embolism. Here, as with deep renous thrombosis, clinical recognition is the exception rather than the rule. Even when an embolus has occluded a major branch of the pulmonary arterial tree, there may be no definite sign to aid in the diagnosis. In our studies of 606 patients with pulmonary cmboli proved at autopsy, a definitive diagnosis was made prior to death in only 7 per cent of the cases. Diagnostic failure was usually due cither to the minimal period of time between onset of symptoms and death or to the obscure nature of the clinical manifestations of the pulmonary embolus. Of those patients in whom pulmonary embolism was thought to be a major factor in producing death, one-fifth died almost inmediately, and almost half of the patients were dead within fifteen minutes. This leaves little time for careful evaluation and the initiation of treatment.

Assessing 501 embolic episodes in 383 patients with significant pulmonary embolism (i.e., embolism which was a major contributory factor or the sole cause of death), it was found that pulmonary embolism withoul infarction was responsible for few if any characteristic signs or symptoms. Pulmonary cmbolism without associated infarction may result in sudden death or there may be shortness of breath, liypotcusion or cyanosis. Chest pain is extremely rare, and, if present, is usually a transitory dull precordial discomfort.

If pulmonary infarction is present, typical or atypical pleuritic chest pain is more frequent, alchough it is still found in less than half of the patients. Hemoptysis occurs only when infarction is present and then only in one patient in five.

A peripheral infarct will occasionally produce a friction rub, but this finding is rare. However, friction rubs are frequently missed because they are transitory. To detect this abnormality the 
chest of a patient with suspected pulmonary embolism must be examined at repeated intervals.

The so-called classic triad of chest pain, shortness of breath and hemoptysis was found in unly 3 per cent of major embolic episudes occuring in our patients.

A sense of awareness of the variable and frequently obscure manifestations of pulmonary embolism should alert the clinician to the possibility that this condition mav exist in a patient with unexplained dyspnea, hypotension or chest pain. The presence of hemoptysis or friction rub should be considered evidence of pulmonary embolism until proved otherwise. Since the manifestations of this disorder are so vague, any suspicious sign is sufficient justification for the immediate institution of aderquate treatment.

Aids in the Prediction and Diagnosis of Thromboembolic Disease

\section{EPIDEMIOLOGIC CONSIDERATIONS}

Certain factors in the history and general medical evaluation may help in making the decision as to treatment of an equivocal case and in the selection of patients for prophylactic thcrapy. Previous studies ${ }^{3}$ have indicated that the incidence of thrombocmbolic disease is much higher when certain epidemiologic factors arc present.

Prior History of Thromboembolic Disease: A prior history of deep venous thrombosis or pulmonary embolism, no matter how remote, is of such ominous prognostic import that we recommend prophylactic anticoagulant therapy in any patient with such a history who requires any significant restriction of activity. Patients in this group with suspicious signs referable to the legs or chest should receive immediate treatment.

Heart Disease: When all patients with heart disease (including those not in heart failure) are considered, pulmonary embolism is three and a half times more frequent in this group of patients than in those without heart disease or cancer. The presence of auricular fibrillation or congestive heart failure increases the incidence about tenfold.

Cancer: Malignant neoplasms of the gastrointestinal tract, lung, or genitourinary tract increase the risk of thromboembolism about fourfold. Other neoplasms have a much lesser effect.

Trauma to the Leg: Bauer ${ }^{4}$ has reported an eightfold increase in thromboembolic complications following trauma to the leg.

Obesity: Extreme obesity is associated with a twofold increase in the risk of thromboembolism.

Immobility: Gilsbs ${ }^{5}$ has shown a direct relationship between the degree and length of immobility and the incidence of thrombeembolic disease. Regardless of the reason for the immobility (operation, fracture, paralysis, serious medical illness), this appears to be a critical factor.

The presence of one or more of these epidemiologic factors should make one more willing to treat an equivocal case in which clinical evidence alone is inadequate to make a definitive diagnosis.

CLINICAL TESTS

Venous Thrombosis: It is our impression that Homans' sign (pain in the calf on dorsiflexion of the foot) is of little or no value in diagnosis because of the high percentage of false positive and false negative results. Tenderness on direct palpation over the course of the deep venous system is much more specific; in the alssence of demonstrable tenderness by this approach, we discount the significance of Homans sign. Measurements of the leg taken at different levels, carefully determined from a fixed point (the superior margin of the medial malleolus), are of real value.

A more oljective evaluation of pain of the calf or thigh may be obtained by slowly inflating a sphygmomanometer cuff placed about the part. Lowenberg ${ }^{6}$ reports that the pressure at which the patient notes pain is considerably lower when renous thrombosis is present. Our experience with this test is limitcd, but it deserves more extensive use to determine its value in detecting subclinical cases of deep venous thrombosis.

Occasionally, in a patient with pain of an equivucal nature in one leg, we have injected intravenously a therapeutic dose of heparin (75 to $100 \mathrm{mg}$.): relief of pain following this procedure is taken as additional suggestive evidence of decp venous thrombosis.

In the past, our laboratory has evaluated a number of tests of various components of the clotting mechanism. None has proved of value, at our present state of knowledge, in detecting the tendency to, or the presence of, thromboembolic disease.

Pulmonary Embolism: Roentgenograms are of little use in the diagnosis of pulmonary embo- 
lism. The roentgenographic picture of pulmonary infarction is variable and non-specific. The so-called classic example of a wedge-shaped infarction is extremely rare. The usual pulmonary infarct is a non-specific pulmonary density which is frequently mistaken for atelectasis or pneumonia. Quite frequently a small pleural effusion is present. It is our belief that a small pleural effusion at the base of the lung with or without a demonstrable pulmonary density should be considered as suggestive roentgenographic eridence of fulmonary infarction unless another definite cause can be proverd.

The electroiardingram is a frequently neglected aid in the diagnosis of pulmonary embolism. Electrocardiographic abnormalities can be found in a high percentage of patients with pulmonary cmboli. Tracings should be obtained early in the course of the discase in order to obtain information of value. Serial records at frequent intervals may be helpful when characteristic electrocardiographic changes are not seen initially and when the diagnosis remains in doub. The so-called acute cor pulmonale type of electrocardiogram is infrequently seen. The more common changes are a shift of the electrical axis to the right, incomplete or complete right bundle branch block, T wave inversion in right percordial leads, RST segment depression in left precordial leads, tall pointed $P$ waves in the limb leads ("P pulmonale") or the onset of an atrial arrhythmia.

While these specific findings often support the clinical diagnosi in certain combinations, may even be strongly suggestive of this condition, the evolution and regression of the electrocardiographic abnormalities are equally important. When these changes result from pulmonary embolism, they usually revert toward normal in a matter of several days or weeks. Tracings taken before the suspected embolism, if available, are valuable for purposes of comparison.

\section{Compilations ind Sequelae of Deep Venous Thrombosis}

Sezere Arteriospasm (Phlegmasia Cerulea Dolens): The problems in diagnosis and the hazards of this complication have already been mentioned.

Pulmonary Embolism: It has been estimated that, without adequate treatment, one patient in three with deep venous thrombosis will have a pulmonary embolus. Of those patients with pulmonary embolism who remain untreated, by the most conservative estimates, at least one patient in five will die from a sccond fatal pulmonary embolus. This means that failure to apply specific therapeutic measures to a patient with deep venous thrombosis will result in an ultimate mortality from pulmonary embolism of at least 6 per cent.

Postphlebitic Syndrome: It is our helief that the physiopathologic process responsible for the development of this syndrome is edema secondary to venous insufficiency. Once persistent edema is present, distention of tissue spaces and thinning of the skin occur resulting from the increased stin tension. The thin edematous shin is more susceptible to trauma and secondary infection. Persistent inflammation is followed by induration, decrease in local arteriolar blood supply secondary to focal scarring and ultimately, ulceration. Bauer ${ }^{i}$ has shown that as the time of olservation after the acute thrombotic episode increases, there is a progressive increase in the incidence of indurative and ulcerative changes. Follow-up studies on patients, five to fifteen ycars after the acute cpisode, have demonstrated an incidence of ulceration of the leg in the range of from 20 to 40 per cent: Bauer has olserved an even higher incidence (all in untreated patients). Zilliacus" has reported that of 689 patients surveved from six to fourtcen years after their thron! yotic episode, only 6 per cent had completely normal legs: 10 per cent were totally incapacitated and unable to work and another 16 per cent had had to change their occupations. These observations indicate that acute venous thrombosis and the sequelae of edema, inflammation, induration and ulceration represent a disabling disease of major magnitude.

\section{Treatment of Thromboembolism}

The tentatze diagnosis of thrombembolic disease is an indication for immediate treatment. The objectives of treatment are to shorten the period of disability and lessen the incidence of complications of deep venous thrombosis. We believe that the optimal treatment is prompt and effective anticoagulant therapy. With this form of treatment both mortality rate and postphlebitic sequelae have been less than during the period when ligation of the femoral vein was practiced.

\section{HEPARIN THERAPY}

Intermittent Intravenous Injection: One may obtain an ahmost instantaneous anticuagulant effect with the intravenous administration of heparin (10 mg. per ml.) in doses of 75 to 100 
mg. More concentrated forms of heparin may result in local burning pain at the site of intravenous injection. Therapy can then be continued with any of several technics. Intermittent intravenous injection of heparin in doses of 75 w $100 \mathrm{mg}$. at eight-hour intervals has been proved by a number of Scandinavian workers to constitute satisfactory therapy, as indicated by a reduction of thromboembolic complications. This regimen provides a "picket-fence" response, clotting times usually returning to normal within five hours after administration of $100 \mathrm{mg}$. of heparin. The alternating return of clotting times to normal values does not appear to diminish the effectiveness of this therapy. Although not absolutely essential, it is wise to check at least one clotting time, just prior to a dose of heparin, to be certain that there is $\mathrm{no}$ cumulative effect from prior doses.

Subcutaneous Injection: An alternative method is the administration of concentrated aqueous heparin (100 to $200 \mathrm{mg}$./ $\mathrm{ml}$.) subcutaneously at twelve-hour intervals. Individual doses vary from 100 to $300 \mathrm{mg}$. The advantage of this method is that heparin may be administered by the nursing staff in hospitals which require that a physician personally administer intravenous medications. The disadvantage is that repeated determinations of clotting time are necessary to adjust dosage to assure adequate effect without risk of hemorrhage. An adequate anticoagulant effect will not be olstained by this approach in a small percentage of patients, presumably because of heparin-inactivating enzymes present in the subcutaneous tissues.

In the past, several heparin preparations incorporated in slowly absorbable menstrua have been used in deep subcutaneous injection. Wc have abandoned these because of the frequency of pain and hematomas at the site of injection and because of a somewhat unpredictable response.

Continuous Intravenous Infusion: In rare instances involving patients with a potential source of major hemorrhage, there may be some advantage to the administration of heparin by constant intravenous infusion. Aqueous heparin, 250 to $300 \mathrm{mg}$., is placed in $1,000 \mathrm{cc}$. of 5 per cent dextrose in water and infused, usually at a rate of 20 to 30 drops per minute, so that there is at least a twofold prolongation of the clotting time. This procedure obviates the extreme prolongation of clotting time which occurs immediately after the rapid administration of a single dose of heparin. Use of this technic requires rigid control of the rate of intravenous infusion to prevent excessive prolongation of the clotting time and the risk of serious hemorrhage.

Bleeding Due to Heparin: One of the major advantages of heparin as an anticuagulant is that its effects on the clotting mechanism may be immediately reversed, in the event of bleeding or if the need for an emergency operation arises, by an intravenous injection of protamine sulfate, usually in an amount equal, milligram for milligram, to the last dose of heparin. It should be remembered that in cases of bleeding following subcutancous administration of heparin, half the protamine should be given by prompt intravenous injection and the remainder by constant intravenous infusion over the period required for ahorption into the blond stream of the heparin remaining in the subcutancous depot.

\section{ORAI, ANTICOAGLLANTS}

Oral anticoagulants provide greater convenience in the management of the usual case of thromboembolic disease. The initial dose of one of the drugs of the coumarin or indandione groups is given at the time of the first dose of heparin. Heparin is continued until the Quick prothrombin activity has reached a satisfactory level. It is esscntial that the initial and subsequent blood specimens drawn for prothrombin detcrminations be obtained at a time when the heparin effect has completely subsided, since some of the extrancous, predominantly physical, effects of heparin may produce falsely low prothrombin activity values. Subsequent doses of the oral anticoagulant are determined on the basis of daily Quick prothrombin activity levels. Except in unusual circumstances, daily prothrombin times should be obtained for all hospitalized patients receiving anticoagulants, regardless of the particular agent being used.

There is considerable variation in opinion as to what constitutes effective therapy with oral anticoagulants. Until recently we have attempted to maintain values between 15 and 30 per cent activity. A statistical study, ${ }^{9}$ however, did not yicld proof that a lesser effect was associated with any higher incidence of thromboembolic complications. For this reason, for the past several years, we have maintained half of our patients treated with anticoagulants in the 30 to 50 per cent Quick prothrom.sin activity range; it is still too early to obtain valid statistical data with regard to the comparative inci- 
dence of bleeding and thromboembolic complications in the two groups.

Advantages of Various Oral Anticoagulants: It is our current opinion that there is no major therapeutic advantage of one oral anticoagulant over another. Our experience with all the clinically available coumarin and indandione derivatives indicates that the thromboembolic complication rate is not significantly lower with one agent than with another. Adjustment of drug dosage to maintain relatively constant prothrombin activity values is easier with some agents than with others. It is preferable to gain extensive experience with one anticoagulant, and this in itself facilitates proper management of dosage. Our own personal preference in treating hospitalized patients is to use a shortacting anticoagulant, such as phenylindandione, which can be given on a twice-daily dosage schedule, or, rarely, once a day in circumstances of extreme drug sensitivity. This system has the advantage that only half of each day's dose of anticoagulant has been given at the time that the daily prothrombin activity report is reviewed. When therapy is to be interrupted for any reason, the return to normal prothrombin activity levels is relatively rapid. The long-acting drugs can be administerce in a single daily dose or as infrequently as once every two to five days. It seems simpler to us to give doses of a drug at 24-hour intervals rather than to gauge dosage by waiting for prothrombin activity values to jump to higher levels. Other investigators believe that the long-acting anticoagulants are best given by a schedule of administration in which a subsequent dose of the drug is given only when prothrombin activity values indicate that the patient is escaping from the desired therapeutic range.

Hemorrhage Due to Oral Anticoagulants: Bleeding due to any of the oral anticoagulants can be controlled by the intravenous or oral administration of vitamin $K_{1}$ in doses of 5 to $50 \mathrm{mg}$. Except in rare instances of hemorrhage secondary to gross overdosage, bleeding will stop within four to eight hours, sometimes less, after the administration of vitamin $K_{1}$. Minor bleeding, when continued anticoagulant treatment is contemplated, is best controlled by decreasing the anticoagulant dosage or by giving small amounts of vitamin $\mathrm{K}_{1}$ (5 to $10 \mathrm{mg}$ ). Larger doses of vitamin $\mathrm{K}_{1}$ may be followed by a prolonged period of refractoriness to the effects of oral anticoagulants.
The incidence of hemorrhagic complications in our series of patients treated with anticoagulants for deep venous thrombosis and/or pulmonary embolism was 14.8 per cent. Most of the episodes were of a minor nature and did not interfere with continuation of treatment. Major bleeding (requiring blood replaccment and or cessation of anticoagulants) occurred in 2.4 per cent of the treatment courses; in almost cvery instance this major blecding took place in an operative wound or at the sitc of a preexisting gastrointestinal lesion. Bleeding was successfully controlled by the regimen described in every instance.

Duration of Anticoagulant Therapy: As yet there is no conclusive information with regard to the duration of anticoagulant therapy required to minimize thromboembolic complications. Analysis of our patients in whom such complications developed during or within twelve weeks after the termination of therapy permits several tentative conclusions. Anticoagulant treatment should be continued until the patient is free of all signs and symptoms and fully ambulatory. Failure to comply with either of these criteria will lead to a higher complication rate. We have also found that added care is necessary in the treatment of patients with heart disease in whom embolic complications are more frequent. Our complete analysis of the effectiveness of anticoagulant therapy has been preriously published ${ }^{9}$; it should be stressed here, however, that fatal pulmonary emboli subsequen to the initiation of anticoagulant treatment were four times more frequent in those patients who had one or more pulmonary emboli before the diagnosis was made and therapy instituted. No anticoagulant will dissolve existent thrombi; the functions of anticoagulant agents are to prevent further propagation of a fresh friable thrombus and to permit organization of the thrombus already present.

\section{PLASMIN AND PLASMIN ACTIVAT'ORS}

There has been considerahle enthusiasm in the past several years concerning the role of plasmin and certain plasmin activators (streptokinase, urokinase) in the treatment of thromboembolic disease. Good objective evidence that these agents reduce either morbidity or mortality from thromboembolic disease is lacking at the present time. Years of competent investigative work and accumulation of statistical data will be necessary before the value of these agents can be determined. There are no 
well controlled studies to suggest that intramuscular or sublingual enzyme preparations have any value whatsoever. A number of laboratories, including our own, have shown that circulating fibrinolytic enzyme has a profound effect on the proteolytic degradation of fibrin and fibrinogen. However, a blood clot and an intravascular thrombus should be considered as separate and distinct entities. The intravenous administration of high concentrations of non-pyrogenic plasmin or plasmin activator to a patient with a thrombus less than seventy-two hours old may subsequently be shown to be an effective and safe method for complete and permanent dissolution of intravascular thrombi; however, until this is conclusively demonstrated by objective technics, these agents should be restricted to clinical investigative use.

VEIN LIGATION

The indication for vein ligation, in our opinion, is the presence of a contraindication to, or the failure of, anticoagulant therapy. Contraindication to the use of cither heparin or oral anticoagulants is limited to those patients in whom the risk of bleeding is so great, or in whom the result of bleeding would be so serious, that the use of any drug which will alter the clotting mechanism is not warranted. Patients who have had recent operations on the eye, brain or spinal cord or with a history of recent ccrebral hemorrhage and those who have had a recent prostatectomy, particularly by transurethral resection, fit into this category.

Progression of venous thrombosis or recurrent pulmonary embolism secms to occur more frequently during treatment with oral anticoagulants than with heparin. When this occurs, heparin therapy should be instituted and, in most instances, no further complications will result. However, if a second complicating pulmonary embolus should occur, operative treatment is recommended.

lena Cava filter: Since the development by DeWeese and Hunter ${ }^{10}$ of a "vena cava filter" we have recommended this procedure as the operation of choice. This technic involves the placement in the inferior vena cava just below the renal veins of one or two rows of interlacing angulated silk sutures which tend to trap any dislodged emboli. It has the advantage of renoving any emboli of significant size from the venous circulation without resulting in complete interruption of caval blood flow, which would occur with ligation. Extensive animal studies and the results in five human subjects with multiple prior pulmonary emboli have shown this to be a safe and effective procedure, not followed to date by the extrcmely disabling sequelae associated with caval ligation. In all five patients on whom this operation was performed the indication was repeated pulmonary emboli, rather than contraindication to anticoagulant therapy, thereforc, it was possible to administer anticoagulants during the postuperative period; this may have helped to minimize thrombosis around the silk suture material until pseudoendothelialization had taken place.

Femoral or lina Caval Ligation: We hasc commented in the past on the fallacy of reliance on superficial ligation of the femoral vin as a measure to prevent pulmonary embolism. As McLachlin and Paterson ${ }^{11}$ have shown, over half of deep venous thrombi are found in locations where bilateral superficial ligation of the femoral vein will not prevent subsequent embolism. Bilateral common femoral or vena caval ligation are more effective but are associated with a much higher incidence of postphlebitic sequelae; in addition, all these procedures have been followed by a significant postligation mortality from fatal pulmonary emboli arising from thrombi propagating just proximal to the site of ligation. It is for these reasons that we restrict our operative procedures to the indications listed herein, and prefer to continue our evaluation of the vena cava filter rather than to carry out ligation per se.

\section{EPIDCRAL SYMPATHETIC BLOGK}

Brief comment has been made previously on additional measures of value in the trcatment of phlegmasia cerulea dolens. If there exists arteriospasm of a degree sufficient to compromise the arterial circulation, immediate treatment is essential. Continuous epidural sympathetic block should be instituted by insertion of a polyethylene catheter into the epidural space with intermittent injection of a local anesthetic for periods of several days to a week or longer. To minimize the possiblity of local bleeding it is essential that the catheter be inserted prior to the beginning of heparin therapy. It is our impression that the latter agent is the preferable anticoagulant because of its apparent beneficial effect in relieving vasospasm. Rapid improvement is usually observed: epidural catheters have been kept in place for as long as ten days but can frequently be removed 
within forty-eight to seventy-two hours while heparin therapy is continued for a considerably longer period.

\section{ANCILLARY MEASURES}

The ancillary non-specific measures used in the treatment of thromboembolism are well known and include bed rest until signs and symptoms subside, repeated careful elastic bandage wrapping of the legs, elevation (6 to 8 inches of the foot of the bed and finally, progressive ambulation with adequately wrapped legs while anticoagulants are still being administered. Antibiotics have no place in the therapy of the uncomplicated aseptic form of deep renous thrombosis. Prophylactic antibiotics may be of value in patients with pulmonary embolism to reduce subsequent secondary infection of pulmonary infarcts.

True septic thrombophlebitis is a separate and distinct disease entity. Pathologically, bacterial colonies are demonstrable in the thrombus. The high frequency of septic cmboli in this condition makes the treatment of choice specific antibiotic therapy and vein interruption well above the site of thrombosis with subsequent anticoagulant therapy to prevent secondary propagation of thrombus proximal to the site of ligation.

\section{TREATMENT OF PULMONARY EMBOLISM}

The therapeutic measures used to treat acute pulnonary embolism seem to have little effect in altering prognosis. The administration of narcotics, oxygen, aminophylline, atropine or papaverine may provide symptomatic relief but they have not been shown to lessen mortality either experimentally or clinically. The use of norepinephrine to treat hypotension secondary to acute cor pulmonale has been shown to alter the mortality rate from experimental pulmonary embolism in dogs ${ }^{12}$ and may have some specific value in man. Unfortunately, fatal cmbolic episodes in our series produced death in almost half of the patients in less than fifteen minutes from the onset of symptoms; this leaves little time in such a group at least for the institution of specific therapy, even if it were available.

\section{PREVENTION AND TREATMENT OF EDEMA}

The treatment of deep venous thrombosis does not end with the acute episode. It is essential that the patient be instructed in a program which will prevent the development of edema, since this is the first and cssential element in the progressive pathologic changes which result in the full postphlebitic syndrome. Our patients are instructed to wrap their legs to the knees with elastic bandages before they get up in the morning and to wear these supports as long as they are on their feet for a period of from six weeks to six months. A trial at full activity without support is then begun; if any edema of his legs develops, the patient returns for measurement and fitting of elastic stockings designed and made to fit his edemafree leg. In addition, these patients are instructed to sleep with the foot of the mattress elevated 6 to 8 inches. Rest during the day with the legs elevated is advised if any discomfort is noted.

It is important that elastic stockings be individually constructed to the patient's own leg measurements. Hydrodynamic studies have shown that valvular incompetence in the recanalized deep venous system results in persistent elevation of venous pressure comparable to the pressure exerted by a column of water reaching to heart level. Pressure is greatest at the level of the ankle and foot and progressively decreases as one moves up the leg. It is important that clastic stockings arc made 10 counteract these pressure relationships by $c x-$ erting the greatest pressure at the ankle area, with progressively less compression further up the leg. The form of the stocking will vary with the contour of the individual leg. If the pressure does not progressively decrease, there may be an area of too great renous compression at one point, which may actually hamper venous return and do more harm than good.

Patients who have carefully followed this regimen from the time of the acute thrombotic episode have been free of advanced skin change and ulceration. Occasionally pain in the leg or fatigue may occur, but the disabling sequelae do not.

\section{PREVENTION OF THROMBOEMBOLISM}

Prior comments on the extreme difficulty and the inaccuracy of the clinical diagnosis of thromboembolic disease were not meant to create an atmosphere of therapeutic nihilism. However, we must be realistic about what we can accomplish, even with maximal clinical diagnostic accuracy, in our therapeutic attempts to lower the disability and mortality from this disease.

Roughly, at the present time, we diagnose 
atrout 10 per cent of the cases of deep renous thrombosis. Another 10 per cent have some localizing sign which should result in the diagnosis of this entity. The remainder, however, have no recognizable clinical signs which may be utiilized for even a presumptive diagnosis. In our series at lcast (substantiated by others ${ }^{13},{ }^{14}$ ), the diagnosis of pulmonary embolism is less accurate. In many instances, the first pulmonary embolus is the fatal one.

Utilizing a critical approach for the analysis of the value of anticoagulant therapy, we have estimated that anticoagulant treatment, as now practiced, will salvage at least scven of every ten patients who would have died from fatal embolism had anticoagulants not been used." Thesc figures were obtained with the assumption that any unexplained sudden death occurring in a patient within twelve wceks after the termination of anticoagulant therapy was due to pulmonary embolism. 'The true rate of salvage with anticoagulant therapy is probably somewhat better than this.

Even assuming that the patients in whom we do make a positive diagnosis represent a higher proportion of those patients with more advanced and more serious forms of thrombocmbolism, it would not scem unreasonablc to estimate that we treat only one patient in cight with fatal or potentially fatal (without anticoagulant treatment) pulmonary embolism. If one is willing to accept these assumptions, then it must be recognized that we will diagnose, treat and salvage only a small proportion of those patients in whom one can expect fatal pulmonary embolism to derclop. Maximal improvement in our diagnostic accuracy might result in a twofold increase in the number of paticnts treated, but even this will represent sallage of only a small percentage of expected fatalities.

Prophylactic Anticoagulant Therapy: However, we have not yet contracted the nihilistic contagion and still regard thrombocmbolism as a theoretically preventable disease. Every attempt should be made to improve diagnostic accuracy and this should result in a slight reduction in disability and mortality. At our present state of knowledge, lacking a satisfactory diagnostic test, the most rational approach to a significant decrease in mortality and morbidity seems to be the utilization of prophylactic anticoagulant therapy in a selected group of patients in whom the risk of thrombembolic complications is greatest.
Utilizing some of the epidemiologic data that have been accumulated, we recommend that the clinician seriously consider the value of using prophylactic anticoagulants in those patients with a high predisposition to thromboembolic disease who must be immobilized in bed. As mentioned earlier, the greatest risk occurs in patients with a prior history of thrombocmbolism, in older people with heart disease or with certain types of cancer, and in any patients with trauma to the leg or extreme obesity. We now recommend prophylactic anticoagulant therapy in all patients with prior thrombocombolism, congestive heart failure or extreme obesily and are planning to extend our inclications to the oiher categories listed herein. The risks of anticoagulant therapy are far less than the incidence of thromboembolic complications in these patients. The results should justify the time and expense sequired to carry out such a program. Until we find methods for the early laboratory diagnosis of venous thrombosis, this seems to be the only available approach capable of effecting a significant reduction in the incidence of this diseasc.

\section{Summary}

The manifestations of thrombombolism are highly variable and frequently obscure. C:linical diagnosis of these entities is difficult, and the majority of cases will not be diagnosed by the means presently available to us. Nevertheless, a heightened awareness of the vagaries of this disease can result in considerable improvement in diagnostic accuracy. Prompt diagnosis on the basis of the slightest clinical suspicion, followed by immediate and adequate anticoagulant therapy, should result in a measureable decrease in thromboembolic complications. However, if one hopes to achicve a profound reduction in incidence of thromboembolism, the only approach presently available is prophylactic rather than therapeutic. The use of measures to increase venous return in patients who are immobilized in bed, plus the institution of prophylactic anticoagulant therapy in a selected group of patients with a high predisposition to the development of thromboembolic diseases, should result in a significant reduction in disability and mortality from this theoretically preventable discase.

\section{REFERENCES}

1. Boyd, A. M., Jefson, R. B., Ratgliffe, A. H., and Rose, S. S, The logical management of chronic ulcers of the leg. Angiology, 3:207, 1952 . 
2. Coox, W. W. Thrombocmbolism. I. Clinicepathologic correlation. (In press.)

3. Coon, IV. W. Thromboembolism. If. Some epideniologic considerations. (In press.)

4. BAEER, G. Thrombosis following leg injuries. Acla chir. scandinav., 90:229, 1944.

5. Giriss, N. M. Venous thrombosis of the lowe limbs with particular reference to bed-rest. Brit. J. Sierg. 45: 209, 1957.

6. Lowenberg, R. I. Early diagnusis of phletuthrombosis with aid of a new clinical test. J. .1. M. A.. 155: 1566, 1954.

7. Batrk. G. A roentgenolugical and clinical study of the sequelac of thrombosis. Acta chir. scandinar.. 86 S Supp. 74: :5-116, 1942.

8. Zit.magies, HI. Thumbu-embolis jukdumens sociola $o c$ l ekonomiska betydelse. Word. med., 37:624, 1) 48 .

9. Com, W. W, Mackenzie, J. W., and Iodgosor, P. E. A critical evaluation of anticoagulant therapy in peripheral venous thrombosis and pul- monary cmbolism. Surg. (innes. Otst., 106: 129, 1958

10. DeWFese. M. S. and Hunter, D). (., JR. A vena cava filter for the preventiun of pulnonary cmboli. Bull. Soc. internat. chir. Brux. 17:17, 1958

11. Molachllin. J. and Palerson. J. C. Some basic observations on venous thrombosis and pulmonary embolism. Surg. Gynec. Obst., 93:1, 1951.

12. Siate, T) and Salisbury, P. F. The experimental production of the pulmonary embolism syndrome and the effects of variations of the systemic pressure on its course. Surg. Gonme of otst. 103:202. 1956

13. Wercir, (. E. and Faxon, H. H. Thrombophlebitis and pulmonary embolism. I. A. M. A., 117: 1502, 1951 .

14. Suma, M. and Penti, A. On thromboembolic cliagnesis in practice and on the need of anticoagulant prophylaxis. Actr. chir. seandinar, 98 : 130,1949 . 